doing much good work. During the past year it prosecuted no few'er than 17,847 cases in the courts. Through its efforts 49, I 8 disabled animals were temporarily suspended from work 34,264 horses, disabled past recovery, were humanely destroyed ; 6444 disabled horses were removed from the streets in an ambulance. The number of prosecutions and other official interferences was larger than in previous years, but it does not follow that cruelty is more common than it was. The increase is due to the greater vigilance of the Society's officers.

IN a paper contributed to the current number of the Journal of the Franklin Institute, Prof. Lewis M. Haupt argues strongly in favour of the construction of a ship canal between New York and Philadelphia, connecting the Delaware and Raritan Rivers. Such a canal would, he maintained, extend the Erie Canal and its benefits to Philadelphia, and open to its manufacturers over I6,000 miles of waterways in the great basin of the Mississippi. It would reduce the distance by water to New York harbour from 240 to about 60 miles, would afford an inside and safe passage to Eastern, Sound, and Hudson River ports ; would develop a large population along the entire route, and so benefit the railroads traversing the district. "In short," says Prof. Haupt, "the effect would be to reduce the rate per mile, as well as to shorten the distance between the two greatest centres of population on the American continent, or, we may say, in the world ; for nowhere else on the globe is it possible by so short and inexpensive a waterway to connect such large populations and so many and valuable interests."

THE Bethlehem Iron Company, Pennsylvania, is to erect at the Chicago Exhibition a full-size model of its I25-ton steam hammer, said to be the largest in the world. It will span the main avenue of Machinery Hall, and will rise to a height of 90 feet. At the last Paris Exhibition great attention was attracted by a similar model shown by the Creusot works, but representing only a Ioo-ton hammer.

BARON VON MUELLER records, in the Victorian Naturalist for February, that, while elaborating diagnoses of new Papuan plants, he was pleasantly surprised to find among the novelties an Antholoma. This genus has hitherto been supposed to be restricted to New Caledonia. The Papuan species is dedicated to Prof. van Tieghem. The denticulation of the leaves, the elongation of the setule of the anthers and the three-celled ovulary already separate $A$. Tieghemi from $A$. montanum. Among the novelties are also Oxalis (Biophytum) albiflora, Sloanea Forbesii, which approaches $S$. quadrivalvis in many respects, but is petaliferous, and Quintinia Macgregori is particularly remarkabie.

A "Treatise on Physical Optics," by Mr. A. B. Basset, will be issued shortly by Messrs. Deighton, Bell, and Co.

THE proper title of Mr. A. E. H. Love's work (included in our list of forthcoming scientific books last week) is "A Treatise on the Mathematical Theory of Elasticity."

Messrs. Nalder Bros. aNo Co. have issued price lists, carefully illustrated, of their electrical testing and other scientific instruments, and of their ammeters and voltometers, resistance frames, \&c.

Messrs. Dulau and Co. have issued Part xvii. of their "Catalogue of Zoological and Palæontological Books." It contains lists of works on Mollusca and Molluscoida.

IN Mr. George S. Carr's letter on the terms "centrifugal force" and "force of inertia" (NATURE, p. 463), in the second sentence of the second paragraph, read "in every case as the reaction to the normal component of the centripetal force" (not "centrifugal").

NO. I 169 , VOL. 45]
AT the meeting of the Belgian Academy of Sciences on March 6, Prof. Spring announced that the late Prof. Stas had left, in an almost completed condition, a long and important memoir describing the results of several further stöchiometrical investigations. It is entitled "Silver," and will forthwith be edited, presumably by Dr. Spring, and published. It may be remembered that, after the publication of Prof. Stas's classical memoir upon the preparation of absolutely pure silver and the atomic weight of that metal, doubts were thrown by Prof. Dumas on the validity of the work on the ground that the silver employed was not free from occluded atmospheric gases. Moreover, Prof. Dumas expressed doubts as to the bearing of the work upon the celebrated hypothesis of Prout, according to which the atomic weights of all the other elements are supposed to be multiples of that of hydrogen. For, if silver possessed the atomic weight attributed to it by Prof. Stas, the atomic weight of oxygen became $15^{\circ} 96$ and not the whole number 16 , and consequently Prout's hypothesis in its original form would be negatived. In order to set these doubts at rest, and to leave his work in a perfected condition, Prof. Stas has prepared a quantity of silver with such extreme precautions that he has succeeded in obtaining it entirely free from occluded gases, and from even the minutest traces of the materials of the vessels employed. So perfect is the purity of this silver that even when heated to the temperature of the melting-point of iridium not a trace of sodium can be detected in the spectrum of the vapour. With this silver he has repeated his former determinations of the atomic weight of the metal, and it is satisfactory to learn that the final number obtained is, as Prof. Stas himself expected it would be, identical with that formerly obtained. Hence, the objection of Prof. Dumas cannot longer be entertained, and the atomic weight of oxygen would indeed appear to be 15.96 and not 16 , for the numbers obtained by Prof. Stas agree so remarkably that an error of four-hundredths of a unit would apparently be out of the question. In addition to this important memoir, Prof. Stas has also left the data of a series of twelve separate determinations of the stochiometric relation of silver to potassium chloride, the materials for which were the pure silver just described, and a specimen of potassium chloride, also prepared with a care and precaution quite in keeping with the rest of the work of the great analyst. The results of these determinations are described by Prof. Spring a; agreeing in a most wonderful manner, and will afford another valuable base to which the atomic neights of many other elements may be referred. Besides these two memoirs, a third is mentioned by Prof. Spring, relating to the spectra of several metals which Prof. Stas has obtained in the purest state in which these metals have ever probably been seen. The whole of these memoirs, consisting of about fifteen hundred pages of manuscript, it is intended to publish forthwith in three separate treatises.

THE additions to the Zoological Society's Gardens during the past week include a Ring-necked Parrakeet (Palaornis torquatus. ठ) from India, presented by Mr. George H. Whitaker; a Grey. breasted Parrakeet (Bolliorhynchus monachus) from Monte Video, presented by Miss Mildred Whitaker; a Roseate Cockatoo (Cacalua roseicapilla) from Australia, presented by Mr. J. S. Gibbons; a Nutmeg Fruit Pigeon (Carpophaga bicolor) from the Torres Straits, presented by Mrs. Fitzgerald; two Pike (Esox. lucius) from British Fresh Waters, pre:ented by Mr. P. F. Coggin; a Mantchurian Crane (Grus viridirostris) from North. China, deposited.

\section{OUR ASTRONOMICAL COLUMN}

Fuzziness of some Variable Stars. - Mr. Cuthbert $G$ Peek has, during the last six years, used his $6 \frac{1}{4}$-inch achromatic for the investigation of the light-curves of variable stars. In 
this month's I'nnwledge he describes some observations of changes in appearance of a few variable stars at different epochs. Three variables - T Cassiopeiæ, R Cassiopeiæ, and S Herculis-have been frequently observed as $(a)$ remarkably well defined, almost planetary, disks ; (b) well-defined stars, surrounded by a more or less dense, ruddy atmosphere; $(c)$ large, woolly, illdefined images, resembling a small but bright planetary nebula; (d) at minimum, in place of the variable, a slight bluish nebulosity. The changes appear to be real, for stars near the places of the variables have been seen clear and sharp when the haziness of the variables was unmistakable. Other stars with regard to which Mr. Peek has made similar observations are S Cassiopeiæ, R Tauri, R Aurigæ, V Cancri, R Ursæ Majoris, S Ursæ Majoris, R Camelopardi, R Boötis, S Coronæ, $\mathrm{R}$ Aquilæ, and S Cephei.

Astronomical Possibilities at Considerable AltiTUDES.-Prof. Pickering, in No. 3079 of the Astronomische Nachrichten, relates some interesting facts in an article on "Astronomical Possibilities at Considerable Altitudes." They are gleaned from observations made at the Boyden Station of the Harvard College Observatory, which is situated two miles from the city of Arequipa, Peru, in latitude $16^{\circ} 24^{\prime} \mathrm{S}$., and longitude $4 \mathrm{~h} .45 \mathrm{~m}$. 3os. W. of Greenwich, and at an altitude of 8060 feet above sea-level. The air there is so clear and steady that 6.5 magnitude stars are picked out by the naked eye with great ease, and, when the moon is not too bright, the eleven Pleiads can always be counted. The nebula in Andromeda forms also a very conspicuous object, " appearing larger than the moon," while, in the I3-inch Clark refractor, "the whole photographic region of the great Orion nebula, first shown in the Harvard photographs of 1887 , is clearly visible to the eye," rendering it the "most splendid object in the stellar universe." The steadiness of the atmosphere is also very. much remarked there, so much so that a scale of steadiness has been adopted. Some of the brightest stars have been noticed to have as many as six complete diffraction rings round them; while around these, when the seeing was denominated as "perfect," twelve rings have been counted. "Boiling" was also found to be sometimes completely eliminated, for, in observing bodies of the solar system with a 13 -inch and a power of 400 , "it was frequently impossible to detect any wavering of the edges of the disk."

The conclusion that Prof. Pickering comes to with regard to the position of future Observatories is that " moderate altitude is a most desirable qualification," while "for transparent skies one must approach the tropics, and for steady seeing one must have an extremely dry climate."

INCREASE OF THE EARTH'S SHADOW DURING LUNAR ECLIPSES. - In a memoir with the title "Die Vergrösserung des Erdschattens bei Mondfinsternissen" (Abhandlungen der math. phys. Classe der k. Sachsischen Ges. d. Wissenschaften, vol. xvii., Lepzig, 189r), Dr. Hartmann published the results of an investigation into the amount by which the earth's atmosphere increases the diameter of the section of the shadow during a lunar eclipse. An abstract of the memoir appears in the annual report of the Royal Astronomical Society, which has just been issued. Since the time of Tobias Mayer (1750) the coefficient 7 has been assunzed to represent this increase, although nothing is known as to the manner in which this quantity was determined. Dr. Hartmann has reduced all the observations of lunar eclipses observed independently by several astronomers during this century, and has deduced the increase of the diameter of the shadow from them. The result of a comprehensive discussion of 2920 observations of the contact of the shadow with well-defined lunar formations is, that the increase of the semi-diameter of the shadow is $48^{\prime \prime} \cdot 62$ for mean lunar parallax. This corresponds to a coefficient of increase $=\frac{\mathbf{I}}{50} \cdot 79$. The result may perhaps be changed $2^{\prime \prime}$ or $3^{\prime \prime}$ by a discussion of new observations, but not more, so it seems desirable that the value of $\frac{1}{50}$ should be used, when required, instead of Mayer's value of $\frac{?}{50}$.

\section{THE NEW STAR IN AURIGA.}

THE new star is rapidly getting more and more difficult of observation in cons =quence of its waning light. There is very little change in its spectrum, and what change there is is not in the direction recorded of Nova Cygni, so it seems pretty

No. I 69 , VOL. 45$]$ clear that the new body was not a hitherto unobserved nebula to begin with.

The Astronomische Nachrichten, No. 3079, contains (at p. Iog) the following communication from Mr. H. C. Vogel, Director of the Astro-physical Observatory at Potsdam, dated February 29 :-

"Although the spectroscopic observations of the Nova in Auriga are not yet concluded-since the star will probably continue visible for some time-I consider it of importance, in the interest of the subject, to communicate my observations made hitherto, and the conclusions drawn therefrom, even though the latter should not in the future be confirmed in all points.

"Concerning, first, the direct spectroscopic observations, I have, on February 20 , observed the Nova with a compound spectroscope of a dispersion sufficient just to show the nickel line between the $\mathrm{D}$ lines. The hydrogen lines $\mathrm{C}, \mathrm{F}$, and $\mathrm{H} \gamma$ appeared bright. Their identification was easy by means of a hydrogen tube in front of the slit. These three lines did not exactly coincide with the lines of the comparison spectrum, but were displaced considerably towards the red, without, however, separating completely from the artificial lines, since they were very broad. The continuous spectı um appeared faint, owing to the comparatively high dispersion; and with certainty only the dark hroad $\mathrm{F}$ line was recognizable, situate towards the more refrangible side, distinctly separated from the bright line in the spectrum.

"Between $C$ and F, a large number of bright lines could be seen, but most of them were too faint to be fixed with certainty. In the case of two brighter lines near $F$, myself and Mr. Frost, who assisted in the observations, succeeded in making very certain wave-length determinations; we found $492.5 \mu \mu$ for the fainter of the two lines, which appeared broad and fuzzy on both edges. and 50I $6 \mu \mu$ for the brighter line. The limit of error is to be taken at about $\pm 3 \mu \mu$, and it results from the observation with certainty that the brighter line is not identical with the double line of the air spectrum or with the brightest line of the nebula, and still less the other with the second nebula line. From Young's list of lines most frequent in the chromosphere, it follows that near $\mathrm{F}$ only the two groups of lines, $501 \cdot 87,501 \cdot 59$, and $493.44,492 \cdot 43,492 \cdot 24,491 \cdot 92$ frequently appear bright. There is no doubt that both line: in the spectrum of the Nova are chromosphere lines, and this result appears to me of great importance. in so far as it is made probable that the line observed in Nova Cygni (1876)-W.L. $500 \mu \mu \pm$ I $\mu \mu$-which, during the gradual fading of the star, alone remained, was a chromosphere line, and not the nebula line.

"Further, both myself and Mr. Frost saw probably the magnesium lines, certainly the sodium lines bright, as also two lines between $b$ and $\mathrm{D}$, one of which probably was the well-known chromosphere line W.L. $531^{\circ} 72$, also observed in Nova Cygni. By direct comparison with the hydrocarbon spectrum, the brightest band of which nearly coincides with the $b$ group, and with the sodium flame, $b$ and D were identified. Mr. Frost could see a displacement of the D lines in the star spectrum with respect to the comparison spectrum. There was no indication of hydrocarbon bands in the spectrum of the Nova.

"Up to the present eleven mostly very good spectrographic photographs have been taken; they were obtained by means of a small spectrograph connected to the photographic refractor of $34 \mathrm{~cm}$. aperture. The dispersion is only small, but in the small spectrum of $10 \mathrm{~mm}$. length, extending from $\mathrm{F}$ to $\mathrm{H}$, much detail is discernible. The illuminating power of the apparatus is so great, in spite of the narrow slit employed, that even now an exposure of 40 minutes is sufficient to obtain an image suitable for measurement. The bright hydrogen lines $\mathrm{F}, \mathrm{H} \gamma, h, \mathrm{H}$, and the calcium line $\mathrm{H}_{2}$, are very broad; and, as aiready announced, the corresponding dark lines of a second spectrum are displaced with respect to the bright lines towards the violet, and in spite of the breadth of the latter, are almost entirely separated. There are still some of the hydrogen lines in the ultra-violet visible, but they are too faint for any approximately certain observation.

"In the last few days the spectrum has changed, inasmuch as in the broad bright lines $\mathrm{H} \gamma, h, \mathrm{H}$, and $\mathrm{H}_{2}$ ( $\mathrm{F}$ is only traced on plates which are over-exposed for the middle of the photographic spectrum), two maxima of intensity are plainly discernible, and, as in each of the corresponding dark lines, a narrow bright line has appeared. From the measurements, a connection between these and the hydrogen lines appears beyond doubt, and it is 